\title{
Positioning long lines: contrast versus plain radiography
}

\author{
A Reece, T Ubhi, A R Craig, S J Newell
}

\begin{abstract}
Aim-To assess the value of contrast versus plain radiography in determining radio-opaque long line tip position in neonates.

Methods-In a prospective study, plain radiography was performed after insertion of radio-opaque long lines. If the line tip was not visible on the plain film, a second film with contrast was obtained in an attempt to visualise the tip.

Results-Sixty eight lines were inserted during the study period, 62 of which were included in the study. In 31 , a second radiographic examination with contrast was necessary to determine position of the tip. In 29 of these, the line tip was clearly visualised with contrast. On two occasions, the line tip could not be seen because the contrast had filled the vein and obscured the tip from view. Eight of the lines that required a second radiograph with contrast were repositioned.

Conclusion-Intravenous contrast should be routinely used in the assessment of long line position in the neonate.
\end{abstract}

(Arch Dis Child Fetal Neonatal Ed 2001;84:F129-F130)

Keywords: long lines; radiography; contrast; cardiac tamponade

Percutaneously inserted intravenous long lines are widely used in the care of preterm and sick neonates. ${ }^{1}$ Care must be taken to insert the line to a satisfactory position before use, usually at the junction between the superior vena cava and the right atrium or immediately above the diaphragm if inserted into the inferior vena cava. ${ }^{2}$ Incorrect positioning can result in life threatening complications including cardiac tamponade. ${ }^{3-5}$ The position of the tip is usually assessed on a plain radiograph. Our policy was to reserve contrast for cases in which the line tip is not visible. The detection of a line on

Accepted 12 October 2000

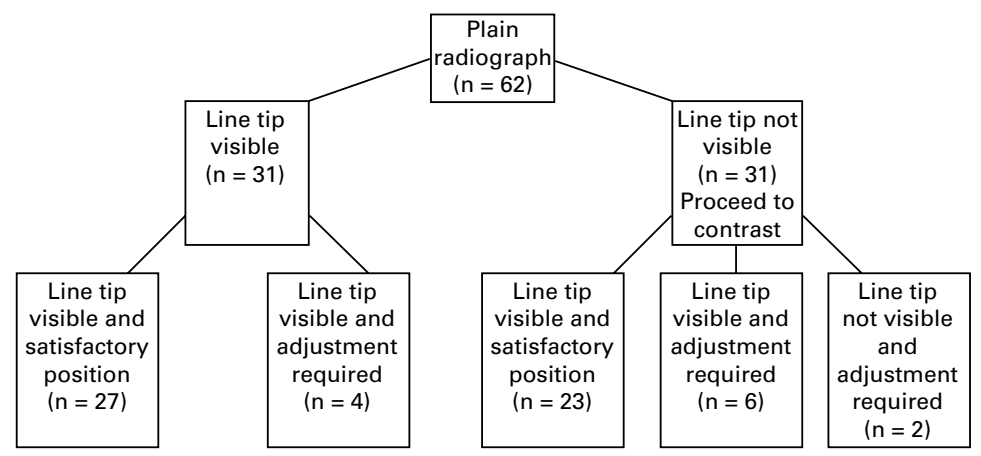

Figure 1 Protocol and results for long line positioning. plain radiography is limited by the radioopacity of the line, the quality of the radiograph, and the experience of the viewing clinician. Therefore, we carried out a prospective study to investigate the role of contrast in the radiographic localisation of the long line tip.

\section{Method}

A prospective study was conducted on a regional neonatal intensive care unit. Over a six month period between August 1998 and February 1999, all infants requiring a percutaneous central venous line were eligible for inclusion in the study. This study was approved by the local research ethics committee. Long lines were inserted according to standard procedure by junior medical staff. ${ }^{2}$ Two types of line were used: the silicon EpicutaneousCava-Katheter and the polyurethene Nutriline (both from Vygon (UK) Ltd, Cirencester, Gloucestershire, UK). The length of the catheter required for correct placement was estimated before insertion using a single measurement of the surface anatomy. After insertion, a plain radiograph was taken to assess the position of the long line tip. We aimed to place the tip of the line up to $10 \mathrm{~mm}$ into the right atrium (upper limb insertion) or in the proximal inferior vena cava (long saphenous insertion). The radiographs were reviewed by the junior medical staff. If the position of the line tip could not be determined accurately, radiography with contrast was performed. A 2 $\mathrm{ml}$ bolus of non-ionic, water soluble contrast medium (Omnipaque; Nycomed Imaging AS, Oslo, Norway) was injected into the long line by a doctor during radiographic exposure. If the line tip position was not satisfactory, it was adjusted accordingly. The plain radiographs (and those with contrast) were reviewed by a consultant neonatologist or radiologist, blind to patient identity and decisions that had been taken at the time of insertion. This allowed validation of the decision taken at the time on the need for a contrast study.

\section{Results}

A total of 68 lines were inserted in 55 babies during the study period. Of these, 62 lines from 49 babies with a median gestation of 29 weeks (range 23-40) and a median birth weight of $1220 \mathrm{~g}$ (range 700-3440) were included in the study. Six lines were excluded from the study (line removal before contrast $(n=1)$, contrast film only $(n=1)$, incorrect documentation $(n=1)$, inappropriate use of contrast $(n=3))$. There was consistent agreement as to whether a contrast study was required between those making the decision at the time of insertion and the senior clinician reviewing the films blind to 

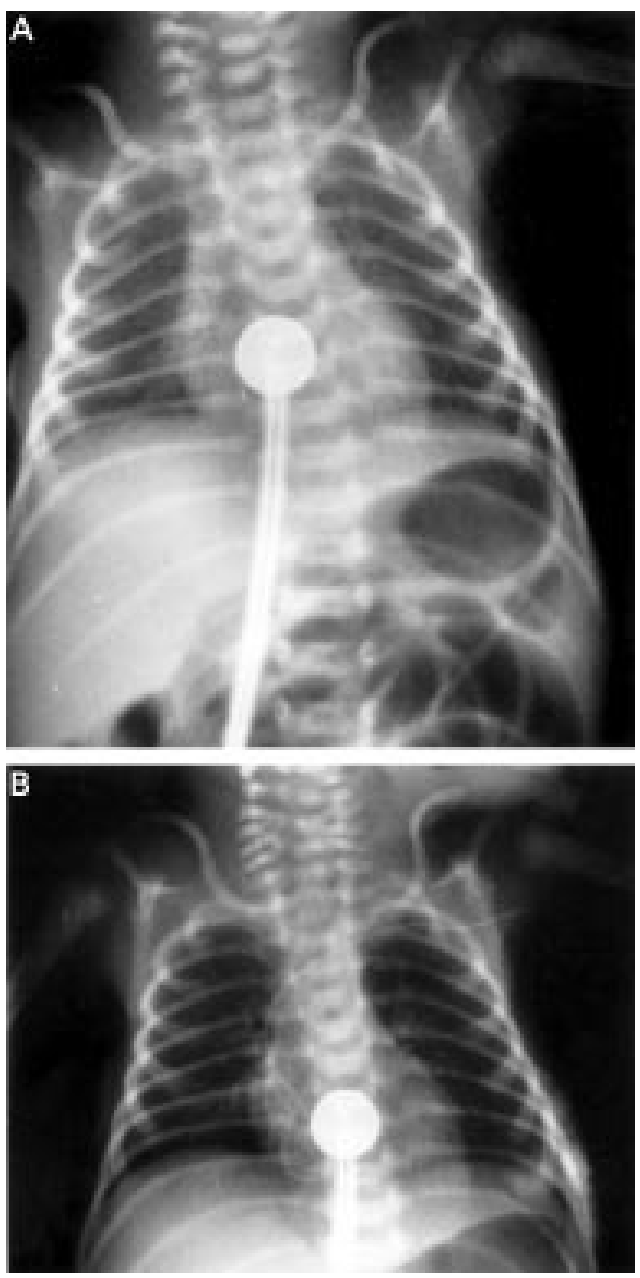

Figure 2 (A) Plain radiograph. It does not show the long line tip with accuracy therefore a film with contrast was taken. (B) The contrast film showing that the catheter is coiled in the heart.

those decisions. There were two lines that the consultant radiologist felt should have been confirmed by contrast radiograph after insertion to confirm the position of the line tip, but which were accepted at the time of insertion on the plain film. No adverse effects were seen in these two cases. In two other cases, the line tip was not visible on the second radiograph after injection of contrast. In both these lines, the line tips were obscured by contrast filling the vein, preventing the line tip from being seen clearly. Both lines were adjusted and the line tip position was checked on a subsequent radiograph. It is assumed that a delay in the radiographer exposing the film allowed more contrast to be injected into the vein thus outlining it and preventing the contrast filled tip from being seen.

The position of $12(19 \%)$ lines required adjustment after insertion. Four of these lines were repositioned after adequate localisation of the tip on a plain film. Eight lines were adjusted after the second contrast film: six where the contrast film visualised the line tip position more clearly, and the two lines with tips obscured by contrast as discussed above. During the study, no infant had adverse effects attributed to long line position or the use of contrast. Half of the lines inserted required a contrast film to visualise the long line tip (fig $1)$.

The plain film can be misleading. In one instance, a contrast film was ordered because the plain film did not show the line position clearly. The contrast film showed the line to be looped in the atrium having been inserted too far (fig 2).

\section{Discussion}

Long line positioning is important in avoiding complications. The narrow calibre silastic line cannot always be seen on plain radiography. To improve radio-opacity, contrast medium is injected into the long line in sufficient volume to fill the connecting device and catheter. The contrast medium was chosen after discussion with the radiologist. Our study did not have the power to exclude adverse effects from the contrast medium, although none were seen.

In our prospective study, we have shown that the position of the line tip could not be accurately determined in $50 \%$ of plain radiographs. This was in a centre where the lines were inserted by experienced junior paediatric staff who made the same assessments of line position as the consultant staff reviewing the radiographs. The judgment of line position on plain radiography may be more difficult for those who do not use percutaneous long lines frequently. The benefits of clear visibility of the line given by the contrast study include not only reducing the potential for complications related to long line use but also halving radiation exposure, reducing the cost, and making better use of medical time. This leads us to conclude that contrast should be used routinely in determining long line positioning at the time of insertion.

We thank Dr Peter Dear, Dr William Ramsden, and the staff of the radiology department and neonatal unit.

1 Brain AJ, Roberton NRC, Rennie JM. Textbook of neonatology. London: Churchill Livingstone, 1999:1376.

2 Brain AJ, Roberton NRC, Rennie JM. Textbook of neonatology. London: Churchill Livingstone, 1999:925-6.

3 Bar-Joseph G, Galvis AG. Perforation of the heart by central venous catheters if infants: guidelines to diagnosis and venous catheters if infants: guidelines to
management. $\mathcal{F}$ Pediatr Surg 1983;18:284-7.

4 Goutail-Flaud MF, Sfez M, Berg A, et al. Central venous catheter-related complications in newborns and infants: a 587-case survey. F Pediatr Surg 1991;26:645-50.

5 Chathas MK, Paton JB, Fisher E. Three years' experience in a neonatal intensive care unit. American fournal of Diseases in Children 1990;144:1246-50. 